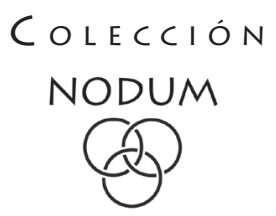


$\because$ Comprometidos con ns 


\section{Desafíos en la formación de psicólogos de las organizaciones y el trabajo}

Johnny Orejuela

-Editor académico-

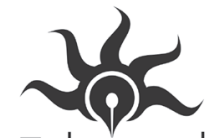

Editorial

EAFIT 
Desafíos en la formación de psicólogos de las organizaciones y el trabajo / Johnny Orejuela, Sigmar Malvezzi, José María Peiró... [et al]; editor académico, Johnny Orejuela. - Medellín: Editorial EAFIT, 2020 362 p.; 24 cm. -- (Nodum)

ISBN: 978-958-720-677-7

ISBN: 978-958-720-678-4 (versión EPUB)

1. Psicología industrial - Enseñanza. 2. Psicología industrial - Orientación vocacional. I. Malvezzi, Sigmar.

II. Peiró, José María. III. Orejuela, Johnny, edit. IV. Ribeiro, Marcelo Alfonso, pról. V. Tít. VI. Serie

$158.7 \mathrm{~cd} 23 \mathrm{ed}$.

D441

Universidad EAFIT - Centro Cultural Biblioteca Luis Echavarría Villegas

\section{Desafíos en la formación de psicólogos de las organizaciones y el trabajo}

Primera edición: diciembre de 2020

(C) Johnny Orejuela -Editor académico-

(D) https://orcid.org/0000-0001-9181-463X

(C) Editorial EAFIT

Carrera 49 No. 7 sur - 50

Tel.: 26195 23, Medellín

http://www.eafit.edu.co/fondoeditorial

Portal de libros: https://editorial.eafit.edu.co/index.php/editorial

Correo electrónico: fonedit@eafit.edu.co

ISBN: 978-958-720-677-7

ISBN: 978-958-720-678-4 (versión EPUB)

DOI: https://doi.org/10.17230/97895872067771r0

Editor: Cristian Suárez Giraldo

Diseño y diagramación: Alina Giraldo Yepes

Imagen de carátula: 1813417270, Coshutterstock.com

Universidad EAFIT | Vigilada Mineducación. Reconocimiento como Universidad: Decreto Número 759, del 6 de mayo de 1971, de la Presidencia de la República de Colombia. Reconocimiento personería jurídica: Número 75, del 28 de junio de 1960, expedida por la Gobernación de Antioquia. Acreditada institucionalmente por el Ministerio de Educación Nacional hasta el 2026, mediante Resolución 2158 emitida el 13 de febrero de 2018

Prohibida la reproducción total o parcial, por cualquier medio o con cualquier propósito, sin la autorización escrita de la editorial

Editado en Medellín, Colombia 


\section{Contenido}

Prólogo

Presentación

Nuevas demandas a la formación profesional de los psicólogos de las organizaciones y el trabajo

Sigmar Malvezzi

Universidad de São Paulo (Brasil)

Ingenuidad y rivalidad paradigmáticas en la psicología organizacional y del trabajo, y sus efectos de (de)formación

Johnny Orejuela, Andrés Vásquez, Milena Villamizar Reyes

Universidad EAFIT (Colombia)

Producción de conocimiento, práctica profesional y formación en psicología organizacional del trabajo: desafíos presentados por el contexto latinoamericano

Erico Rentería Pérez, Adriano de Lemos Alves Peixoto,

Antonio Virgilio Bittencourt Bastos

Universidad del Valle (Colombia), Universidad Federal de Bahía -UFBA- (Brasil) 
Investigación científica y práctica profesional en

la psicología organizacional y del trabajo:

estrategias desde la formación

Juan Pablo Gamboa Navarro, Carmen Picazo Lahiguera,

María Constanza Aguilar Bustamante

Orkestra-Fundación Deusto y Deusto Business School, Universidad de Deusto (España)

Universidad Loyola y Universidad de Zaragoza (España), Universidad Católica de

Colombia (Colombia)

La identidad de carrera y la formación de psicólogos organizacionales

Juan Javier Vesga

Universidad El Bosque (Colombia)

Retos actuales y necesidades de la formación en psicología del trabajo: reflexiones desde la artesanía

Perla Shiomara del Carpio Ovando, Marta Romero Delgado

Universidad de Guanajuato (México), Universidad Complutense de Madrid (España)

Competencias para la formación en psicología organizacional y del trabajo: reflexiones a partir de una investigación sobre el trabajo en un contexto de conflicto y desventaja social

Gustavo Peralta Hernández, Merlin Patricia Grueso Hinestroza,

Concha Antón Rubio, Mónica López-Santamaría

Universidad del Rosario (Colombia), Universidad de Salamanca (España)

Retos de la economía popular y la inclusión social:

un nuevo escenario de formación profesional desde la psicología del trabajo

Yair González, Leny Sato

Universidad Piloto de Colombia (Colombia), Universidad de São Paulo (Brasil) 
Apuntes sobre la formación de los profesionales en psicología organizacional y del trabajo frente al coaching... 203 Jean David Polo Vargas, Kelly Arroyo Velandia, Milton Zambrano Curcio Universidad del Norte (Colombia)

La formación de los psicólogos del trabajo y las organizaciones con una visión responsable: una formación con perspectiva de género

María Dolores de la Cruz Fresneda

Miembro del Col-legi Oficial de Psicologia de la Comunitat Valenciana

-COPCV- (España)

La subjetividad y la eficacia sindical en la formación de psicólogos del trabajo y las organizaciones

Francisco Mesa Burgos, Tania García Ramos

Universidad de Puerto Rico (Puerto Rico)

Las variables psicológicas positivas como ejes de formación y actuación profesional en el contexto de la salud en el trabajo

Leonor Emilia Delgado Abella

Universidad El Bosque (Colombia)

La formación del psicólogo organizacional y del trabajo en los escenarios laborales actuales

Iván David Jojoa Arcos, Dianne Sophia Araújo Romero

Grupo ConCiencia Human Group Investigación y Consultoría (Colombia)

Desafíos para el docente formador de psicólogos del trabajo y las organizaciones derivados del entorno laboral contemporáneo

Milena Villamizar Reyes, Silvia Morales Tobón

Universidad EAFIT (Colombia), Universidad de San Buenaventura (Colombia) 
Epílogo. Nuevas exigencias formativas del futuro psicólogo del trabajo y de las organizaciones............................ 315

José María Peiró

Instituto de Investigación en Psicología de los Recursos Humanos, del Desarrollo Organizacional y de la Calidad de Vida Laboral (Idocal)

de la Universidad de Valencia (España)

Sobre los autores 
A todos nuestros estudiantes del campo de la psicología del trabajo y las organizaciones, de todos los niveles de formación y todas las generaciones, quienes tienen el desafío de continuar nuestro campo y enriquecerlo, reivindicando cada día, en la cotidianidad, el lado humano, subjetivo, del trabajo y las organizaciones.

A todos nuestros maestros del campo de la psicología del trabajo y las organizaciones por inspirarnos y trasmitirnos la pasión por esta subdisciplina que se ocupa de investigar para trasformar, en pro del bienestar de quienes trabajan, nuestros sujetos de estudio e intervención como ciencia y profesión. 



\section{Agradecimientos}

En primer lugar, nuestros agradecimientos a la Universidad EAFIT, por su confianza en este proyecto y su respaldo para llevarlo a cabo.

Al apreciado profesor Marcelo Ribeiro, de la Universidad de São Paulo, por la generosa e impecable escritura del prólogo, y por secundar siempre nuestros proyectos editoriales.

A todos y cada uno de los colegas del Nodo de Psicología Organizacional y del Trabajo (POT) de la Red Colombiana de Investigadores de la Asociación Colombiana de Facultades de Psicología (ASCOFAPSI), por su disposición para participar en este proyecto, mantener viva nuestra red y enriquecer el campo de la POT en nuestro país con este libro sobre la formación de psicólogos.

A Sigmar Malvezzi y José María Peiró, grandes maestros de nuestro campo en Iberoamérica, por participar de esta iniciativa y por legarnos la mejor formación posible en el campo de la POT.

A todos y a cada uno de los coautores internacionales, quienes se dispusieron a apoyar académicamente esta iniciativa editorial, para estrechar lazos a través de acciones colaborativas en pro del desarrollo de la psicología del trabajo y las organizaciones.

A los coautores de este libro, tanto nacionales como internacionales, que se dispusieron a apoyar financieramente este proyecto, para concretarlo de manera diligente.

Al equipo de la Editorial EAFIT, en especial a su directora, Claudia Ivonne Giraldo, por su apoyo y diligencia; a Cristian Suárez Giraldo por la coordinación editorial; a Liliana Echeverri por la corrección de estilo y a Alina Giraldo Yepes por su trabajo de diseño gráfico.

Johnny Orejuela

Editor académico 



\section{Prólogo}

Escena 1: Mundo contemporáneo. Escenario 1: Mundo del trabajo y organizacional actual. Escenario 2: Mundo de las universidades. Campo principal: La psicología organizacional y del trabajo (POT) con sus personajes centrales: profesionales, investigadores/as, profesores/as, alumnos/as, agregados/as y todos/as aquellos/as que trabajan. Todos/as con el mismo discurso del cambio continuo, de la flexibilización y del tiempo acelerado que generan constantemente desafíos y riesgos de los más variados tipos y de las más variadas dimensiones.

Una importante advertencia es que se habla mucho sobre el futuro del profesional dominado por la inteligencia artificial, mas no se puede olvidar que en el contexto latinoamericano la gran mayoría de los que trabajan lo hacen en condiciones precarias, con actividades repetitivas, vaciadas de sentido y sin la debida regulación o protección social -el contexto importa-, como el presente libro pone en evidencia.

La percepción de la necesidad de cambiar su forma de pensar, actuar y formar mediante sus teorías, prácticas y estrategias formativas se viene intensificando entre los profesionales y teóricos de la POT desde las últimas décadas del siglo XX. En busca de respuestas, muchos procuran renovar el campo, atravesando caminos innovadores o creando nuevos caminos.

Desafíos en la formación de psicólogos de las organizaciones y el trabajo es un libro que mueve al lector hacia la radicalización de la reflexividad, como diría Anthony Giddens, y la posibilidad de enfrentar el mundo por medio de la construcción de nuevas formas de pensar y actuar, saliendo de una POT enraizada en el siglo XX, para entrar en una POT dirigida hacia el siglo XXI, sin la pretensión ni la ingenuidad de pensar que ahora todo es diferente. ¿Podríamos, tal vez, hablar de una continuidad con rupturas y fisuras? Nuevas demandas del mundo del trabajo, la rivalidad paradigmática generadora de deformación de la identidad 
de carrera en POT, la investigación, la producción y las prácticas como estrategias de formación; la cuestión de la inclusión social, la formación como productora de subjetividades, los antagonismos y las interfaces con el coaching, el papel del sindicato, la emergencia de la psicología positiva, entre otras cuestiones, constituyen algunas pizcas de lo que se ofrece en este libro. Pero hay mucho más.

Tanto los/as autores/as como los capítulos de este libro, enfocados en la formación para el campo de la POT, llaman nuestra atención debido a la necesidad de estudiar el comportamiento de los mercados, de analizar la sociedad como un campo de fuerza dentro del cual el/la psicólogo/a tiene que posicionarse y ayudar al posicionamiento de los otros, o entender si la subjetividad digitalizada sigue siendo la misma subjetividad de la psicología clásica.

Nos alerta que hoy tengamos acceso a todo tipo de formación, en calidad y cantidad; parece que cuanto más acceso tenemos a la información, más estamos desinformados/das: ¿quién forma a quién?, ¿quién está socialmente legitimado para formar?, ¿quién o qué garantiza nuestra seguridad ontológica, sin la cual no podemos tener una vida laboral con sentido?, ¿̇cómo lidiar con nuestro sesgo o ceguera paradigmática?, ¿̇cómo no estar de acuerdo con que el contexto importa y la desigualdad social existe y moldea nuestro pensamiento y nuestras prácticas?, ¿cómo evitar las promesas salvadoras generadas por la industria de las consultorías y parte de las prácticas de coaching que se incluyen en la formación en POT?, ¿̇cómo desconsiderar las teorías de género, que señalan que hombres y mujeres son diferentes y merecen serlo, pero que deberían ser iguales en derechos, aunque no lo son?, ¿̇cómo intentar eliminar la brecha entre la investigación y la práctica, lo que es una tarea constante en todos los campos de la ciencia y en profesiones como la psicología?, ¿̇cómo la POT puede dejar de ser una psicología del empleo y alcanzar la categoría de psicología del trabajo? Son muchas las inquietudes, algunas más divulgadas, otras aparecen como propuestas incipientes de los/las autores/ras del presente libro, que tratan de enfrentar la misma cuestión disparadora e integradora. En fin, ċcómo formar a un/a profesional, investigador/ra, formador/ra, y trabajador/ra para el campo de la POT?

Como profesional, investigador/ra, formador/ra, de la POT, Desafíos en la formación de psicólogos de las organizaciones y el trabajo te posicionará mejor en el área de los retos formativos postulados a través de los potenciales insights que serán generados y de la ampliación del espectro de temas y caminos posibles para enfrentar el híbrido, flexible y cambiante mundo organizacional y del 
trabajo del siglo XXI. En cuanto a este panorama, solamente la ampliación, la diversificación y la reconstrucción del campo permitirán enfrentar sus dilemas y oportunidades. Y esta reconstrucción debería evitar ser solitaria, sin cooperación y sin un proceso de coconstrucción. Pues solamente así la radicalización de la reflexividad y el pensamiento divergente generarán innovaciones. Este es un libro que ciertamente abrirá puertas para emprender tal travesía, y ofrecerá las herramientas para lidiar con los constantes y diferentes proyectos que emerjan en ese caminar. iBuena inspiración y buena lectura!

Marcelo Alfonso Ribeiro

Profesor asociado Instituto de Psicología Universidad de São Paulo (Brasil) 



\section{Presentación}

La investigación y la reflexión sistemática sobre las múltiples implicaciones y los desafíos que impone la formación de psicólogos es una tarea aún pendiente en nuestro país, y en general en América Latina. Las implicaciones académicas, éticas y subjetivas del ejercicio de formación de psicólogos son un asunto de obligatoria reflexión e investigación por parte del amplio cuerpo de docentes, a muchos de los cuales les pagan por hora cátedra; pues la formación es en sí misma una práctica, y la docencia universitaria en psicología, una ocupación de por lo menos el 10\% de los psicólogos graduados de nuestro país. Adviértase con conciencia autocrítica que la gran mayoría de los que ejercen la docencia efectivamente en la cotidianidad de las aulas universitarias de nuestros cursos de psicología no tiene formación en pedagogía ni didáctica universitaria, pese a que muchos de los sistemas de selección y escalafonamiento de los docentes universitarios hoy piden por lo menos un diplomado en docencia universitaria.

Existen, además, algunas especializaciones y maestrías, generalmente ofrecidas por facultades de educación, en docencia universitaria -genérica-, que una minoría entre los psicólogos-docentes ha cursado. La tarea está aún pendiente. Los desafíos de la formación de psicólogos en el contexto actual no son menores: el cambio y las brechas generacionales, la presencia y el impacto de nuevas tecnologías de la información, las exigencias de mayor investigación, la emergencia de una naciente revolución tecnológica denominada cuarta revolución industrial, fenómenos de creciente globalización, diversificación y complejización del panorama social, histórico y cultural, el cambio climático, en el ámbito más general, y la asistencia a un momento de transición sociopolítica denominado posconflicto o posacuerdo, en el ámbito nacional particular, son condiciones de posibilidad para pensar que estamos ante un escenario reconfigurado en estos albores del siglo XXI. Este conjunto de cambios desafía a la psicología como 
ciencia y como profesión, para que responda a nuevas demandas de explicación del comportamiento humano, de las nuevas psicopatologías y de la configuración de la subjetividad, entre otros aspectos. Este nuevo escenario desafía a repensar las prácticas de formación, así como los contenidos, las competencias, las formas y el sentido de la evaluación, la interfaz entre teoría y práctica profesional, las formas de trasmisión y evaluación de la formación, así como la pertinencia de los profesionales de cara a las nuevas exigencias del mercado de trabajo. Como vemos, enfrentamos un panorama complejo que demanda recursividad académica, investigativa y pedagógica para asumir el oficio de formar en psicología.

Ahora bien, en el campo específico de la psicología como ciencia, atestiguamos la emergencia de nuevas líneas de pensamiento (psicología positiva), nuevas formas de aproximarnos a la realidad (etnografía digital) e implementar la psicología como profesión (ciberpsicología), nuevos temas de investigación (felicidad, empresas saludables, placer y sufrimiento en el trabajo); y una mayor aceptación de formas de hacer ciencia que desbordan el paradigma dominante, dan lugar a debates más abiertos, aunque no superados en la práctica, y permiten que se desplieguen estudios en una línea más cualitativa y crítica, y que estos sean cada vez más publicados. Una conquista epistemológica para la disciplina.

La dimensión paradigmática característica de la psicología y los múltiples campos de ampliación, así como la combinación de estos aspectos, complejizan aún más la reflexión sobre la formación en psicología, pues cada paradigma tiene un conjunto de presunciones y cánones sobre cómo hacer ciencia, a la vez que cada enfoque teórico concibe de manera específica un objeto de estudio e intervención consecuente, y que cada campo de aplicación demanda formas de intervención ajustadas y consecuentes con sus propósitos profesionales, científicos y técnicos. Paradigmas, enfoques teóricos y campos de aplicación coexisten y se recombinan para producir una profundización de la naturaleza del campo de la psicología. Este es otro asunto a considerar de cara a lo que representa una formación de calidad de nuestros futuros psicólogos.

La psicología del trabajo y las organizaciones como campo de investigación y acción profesional específico no está al margen de tales complejidades y, además, está en interfaz constante con los cambios sociohistóricos, en general, y en el orden productivo y la dinámica del capitalismo, en particular, que le imponen también, desde hace ya cuarenta y cinco años, repensarse de cara a un mundo del trabajo reconfigurado bajo la impronta posfordista: el trabajo por 
proyectos, las organizaciones flexibles y horizontales, las nuevas patologías laborales, la fragmentación precaria del mercado de trabajo, el migracionismo laboral y la presencia masiva de fenómenos de diversidad, y concomitantemente, las demandas ethopolíticas de inclusión social de la diferencia, así como la emergencia de nuevos temas, como la felicidad en el trabajo; o nuevos campos subdisciplinares, como las clínicas del trabajo o la psicología clínica laboral. Por lo menos en nuestro contexto latinoamericano, se imponen nuevos retos que se debe privilegiar o incorporar en la formación de los psicólogos del campo de la POT, de cara a la actualización curricular, pedagógica y temática.

Al terminar este libro comenzamos a vivir la crisis planetaria producida por la pandemia de la covid-19, crisis que sin duda va a tener un impacto en el mundo del trabajo y en la estructura y la función de las organizaciones laborales; este fenómeno contextual no puede ser eludido, pues toca nuestro objeto directo de estudio. Creemos que la ecuación sujeto/persona-trabajo-organización-contexto, aludido por muchos de los trabajos aquí reunidos, evidencia la importancia del contexto y su influjo sobre nuestro objeto de estudio. No abriremos un debate específico sobre el impacto de la pandemia, más bien confiamos en que se ofrecen las herramientas conceptuales para que la reflexión sobre el mundo pospandemia que tendremos sea posible a partir de lo que se plantea en el libro.

Por todo lo anterior, es perentorio para el campo de la POT pensar en las implicaciones y los nuevos desafíos que enfrenta, en cuanto proyecto de formación científico-profesional. Este libro es un granito de arena que aporta en esa dirección. Los invitamos a leerlo y debatirlo, con los autores, los colegas y los propios estudiantes. Que el presente volumen sea solo una excusa para abrir y animar un debate que no puede seguir pendiente ni cesar en el país.

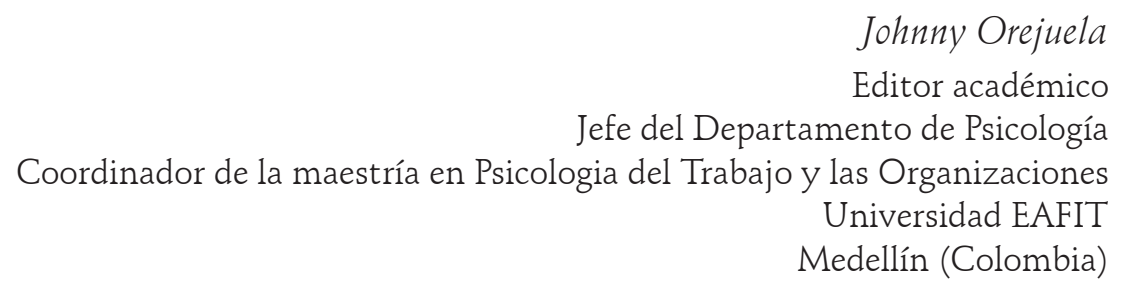

